\title{
RAPID-SURVEY METHODS FOR FLOWERING PHENOLOGY
}

\author{
Lyn Blades ${ }^{l}$, Geoffrey Harper ${ }^{l}$, Stephan Helfer ${ }^{l}$, Maria Luisa Lee ${ }^{l}$, Liz Rogers ${ }^{l}$ \\ \& Sandra Stewart ${ }^{1}$
}

\begin{abstract}
The Weekly Phenology Project at the Royal Botanic Garden Edinburgh has been running for three and a half years at the time of writing. Insufficient data have been collected so far for scientific analysis, but we present provisional conclusions concerning several methods of monitoring flowering behaviour. These range from objective methods, such as presence/absence of open flowers and counts of flowers or inflorescences, to non-objective methods involving assessment of the degree to which full flowering has been achieved. One monitoring method, relying on the relative numbers of flowers in the three states 'bud', 'open', and 'gone-over', is illustrated by the flowering behaviour of 'simultaneously' and 'sequentially' flowering species. We conclude with a discussion of the relative values of the different methods.
\end{abstract}

\section{INTRODUCTION}

Articles on this subject almost invariably begin with a definition of the term 'phenology' - 'the study of seasonal events, especially in organisms and in relation to the weather'. Or, as a Ranger at the Royal Botanic Garden Edinburgh (RBGE) recently offered in haste to a visitor, phenology is 'when stuff happens'.

The current Phenology Programme at RBGE began in January 2002, resuming where daily observations were discontinued in 1895. Then in 2005 a project based on weekly observations was established, in 2007 a Rhododendron Project was added, using methods similar to those of the Weekly Project, and in 2008 cloned material was acquired from Germany to set up three International Phenological Gardens.

During this brief period the purpose of the Phenology Programme has undergone some changes. From an initial aim simply to continue collecting first-flowering dates, as had been done in the 19th and 20th centuries, it has become clear that, if we are to understand how weather affects the timing of flowering, we also need to understand how the whole life cycle is related to environmental conditions and triggers. Ideally we should like to study a wide range of species in depth, with respect to all important aspects of the life cycle, including germination, transition to maturity, onset of dormancy, bud burst,

${ }^{1}$ Lyn Blades, Maria Luisa Lee, Liz Rogers and Sandra Stewart are Phenology Project volunteers at the Royal Botanic Garden Edinburgh.

Geoffrey Harper is a Research Assistant in Phenology at the Royal Botanic Garden Edinburgh. Email: g.harper@rbge.org.uk Stephan Helfer is a Plant Pathologist in the Science Division at the Royal Botanic Garden Edinburgh. Email: s.helfer@rbge. org.uk

Address: Royal Botanic Garden Edinburgh, 20A Inverleith Row, Edinburgh EH3 5LR.

All correspondence to Stephan Helfer at the above address. 
flowering, fruiting, growth, storage, leaf fall, and other phenological events and stages. Sadly, this is impractical.

At RBGE, instead of intensive studies of this kind, we are initially conducting extensive surveys of a wide range of plants in the Living Collections at the Inverleith site (Harper et al., 2004). Typically, volunteer observers walk around a set route in the Garden to monitor about a hundred accessions (individual trees or shrubs, or patches of herbs) spending no more than a minute or so on each one. Maps are provided showing the locations of the accessions and a suggested route (where it does not vary through the year, as in the Daily Project), and recording forms are designed to have one or more spaces for each accession on each date, for noting down the condition of the plant. For instance one space may be used to enter a symbol representing the state of flowering, and another to record flowering using a different method of scoring or to record the state of the foliage. After each monitoring session the data are transferred to an Excel spreadsheet for storage and later analysis.

Rapid surveying of this kind is 'pencil-and-paper' science, in which valuable scientific data are collected using a minimum of equipment at very little expense. On a wet and windy day, it is quite enough to be clutching an umbrella and writing down notes without having to cope with more equipment, especially when the observer is working alone. The only equipment the observer need carry, apart from the umbrella on wet days, is a pencil and clipboard with papers and transparent plastic cover securely attached.

During the first three years of the Weekly Project we have been developing the most appropriate rapid-survey techniques for the plants we are studying. The initial hope was that a single method would be found for all plants. This would have enabled semi-quantitative information to be gathered sufficient for an accurate, if simple, representation of the course of flowering, including the timing of mass and/or peak flowering. That hope was soon dashed. The article describes the pros and cons of the three main methods we have tried using - none of which is, unfortunately, suitable for all plants.

We would encourage anyone interested in the effect of weather and climate on plants to make their own observations - be it in their own gardens, in local urban parks and streets, at school, or on regular walks in the countryside - and in this context we hope the following notes will be of use. It is the kind of project that an enterprising school could conduct over many years, with the observations made by successive generations of students.

At RBGE we intend to analyse the data, when enough have been collected, with a view to identifying species of particular interest and devising hypotheses to explain any patterns in the data. It may then be possible to design experiments to test our hypotheses. We shall also attempt to draw up a classification of functional phenological groups to enable prediction of how any classified species will react to any kind of climate change. In this way we hope to make a serious contribution to the science of plant-climate interactions.

In the RBGE Weekly Project LB, LR, ML, \& SS make the observations, GH does the paperwork, and $\mathrm{SH}$ is the project manager. 


\section{FLOWERING CRITERIA}

As in all science we would like to make objective observations. Here 'objectivity' means consistency in the observations made by independent observers recording the same phenomenon. It would be easy to conduct formal tests of the methods discussed below, but these have not been carried out so far. Instead we offer an informal assessment of the relative objectivity of each method.

The main requirement of an objective criterion of flowering is that the phenological event being recorded should involve observable change which is both rapid and irreversible. Visibility of anther and/or stigma is the standard criterion for a flower having opened, and in many species each flower opens rapidly (in relation to the frequency of monitoring) and only once. Some species, for example Eranthis hyemalis and Anemone blanda, may open so slowly that it is difficult to assign a unique first-flowering date in daily monitoring, although this is not usually a problem in weekly monitoring. Some species may open and close repeatedly according to very recent weather conditions (such as Eranthis hyemalis), and for these the anther/stigma criterion is rather useless. Instead it may be possible to use a height criterion, recording the date when the Crocus or Colchicum flower reaches full or nearly full height; or a position criterion, such as the date when a Galanthus flower bud is first seen 'nodding' (below horizontal) or an Eranthis flower bud is first seen 'erect' (above horizontal).

In some species the anthers and stigmas are not normally visible at all, for example Corydalis and members of the pea family Fabaceae. In this case divergence of the keel and standard petals usually occurs rapidly and provides a satisfactory criterion.

A further problem arises in species which may begin flowering with a few sporadic and sometimes deformed flowers, such as Forsythia $\times$ intermedia and some Tilia species. In this case it makes sense to base the first-flowering criterion on the first date when 5 , or 10 , flowers are open.

End of flowering might be thought to pose far worse problems, since flowers often appear to deteriorate rather slowly, though admittedly usually irreversibly. In fact, though, we have experienced few problems. The homely approach of asking the question, 'Would you pick that flower and put it in a glass of water on your kitchen windowsill?', serves surprisingly well. In other words it is usually clear when an individual flower has 'gone over'. It has begun to wilt, become discoloured or shed parts, or in general no longer looks fresh. End of flowering is recorded when no more fresh flowers are present.

A few flowers have persistent petals, remaining long after the flower is clearly in fruit as some Helleborus species do. In others the petals may be insignificant, such as Hedera and Fatsia in the Araliaceae. In all these cases the stamens fall off rather obviously at the end of flowering, and this provides a good criterion. In the case of some Daphne species the whole flower falls if not fertilized, and this can be used where there is no fertilization and the flowers do not deteriorate markedly before dehiscence. In Digitalis the corolla conveniently falls off to mark the 'gone-over' phase. 
When selecting a criterion the exact stage of development is not crucial. It is more important to (a) choose a criterion which is believed to be objective, (b) describe and record it in writing so that observers and future users of the data know how they were collected, and then (c) stick to the same criterion consistently. Any change of criterion must be clearly recorded to avoid data collected using different criteria being unwittingly combined in analysis.

\section{'BFG' METHOD}

We first describe a method based on the relative numbers of flowers in the bud (B), open ( $\mathrm{F}$ - for 'flowering') and gone-over $(\mathrm{G})$ states. To the best of our knowledge it was developed by E.P. Jeffree $(1951,1957)$ in his studies of pollen and nectar sources for honey bees. For short we call it the 'BFG' method.

Fig. 1 shows the progress of flowering in an ideal individual plant, most of the flowers of which are open simultaneously at peak flowering. Such a species is referred to as 'flowering simultaneously'. It is assumed that all the flowers - technically 'floral shoots' (Glover, 2007) - are in bud form before the first one opens, when $B=100 \%$. When the last flower opens, the number of buds is zero, i.e. $\mathrm{B}=0 \%$. So the B-curve in the diagram - which we call a 'Jeffree diagram' - falls from $100 \%$ to $0 \%$ during the early stages in the flowering period. Correspondingly the G-curve records the number of gone-over flowers on each date, and it of course rises from $0 \%$ to $100 \%$ when the last flower goes over, assuming all buds open.

Since the sum of the B, F and G floral shoots remains the same in our ideal plant, assuming that none is lost through damage, any one quantity can be calculated from the other two. This can be useful when one of the parameters cannot easily be observed. For instance, in the Foxglove example described below, the youngest buds were not visible at the start of the flowering period, but the B-curve could be calculated from daily

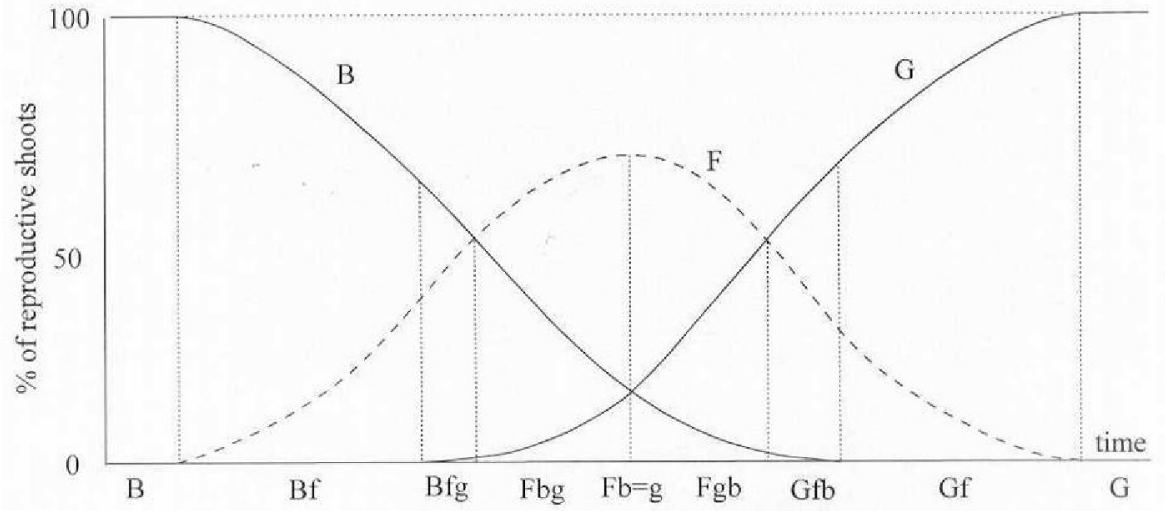

Fig. 1 'Jeffree diagram' showing the relative numbers of buds (B), open flowers (F) and gone-over flowers (G) at each stage during the flowering of an ideal plant. 
counts made of the F and $\mathrm{G}$ flowers. (In our ideal plant the F-curve approximates to a normal probability frequency-distribution curve, although there is no particular reason to suppose any individual plant will have an F-curve of this exact shape.)

The B- and G-curves represent phenological events, namely the transition of each floral shoot from $B$ to $F$ and then from $F$ to $G$ state. The relative timing of these two events determines how long each individual open flower lasts, and the relative timing of the B-and G-curves determines the shape and height of the F-curve. If the G-curve is imagined shifted to the left, meaning that individual flowers do not last so long, the B- and G-curves will overlap more, and the F-curve will be lower. With a great deal of overlap only a small proportion of the flowers will be open at any one time, and in this case we say that the plant flowers 'sequentially'. The Foxglove is an example.

Since making the regular counts needed to construct a Jeffree diagram is labourintensive and by no means a rapid-survey method, we have tried developing a rapid-survey technique based on the relative numbers of B, F and $\mathrm{G}$ flowers. For instance on one day it might be obvious, at a glance, that a plant has mostly buds, with only a few open flowers, and none gone over, and in this case it would be recorded as ' $\mathrm{Bf}$ '. The order of the letters indicates the relative quantities, from most to least numerous. A plant with 50 buds, 10 open flowers and 1 gone-over would be 'Bfg'. (The capital initial letter conveys no information: it merely indicates the start of the record, and this is useful if symbols are squashed together on a recording sheet with no space between them.) Where there are equal numbers of flowers in two states, for instance 80 buds, 10 open flowers and 10 gone-overs, the record would be 'Bf=g' or ' $\mathrm{Bf}: \mathrm{g}$ ', with the '=' or colon signifying equality or situations where it is difficult to tell which of two states is more numerous. In Fig. 1 the mid-point (and peak flowering) is represented by ' $\mathrm{Fb}=\mathrm{g}$ ', since there are equal numbers of B and $\mathrm{G}$ floral shoots.

Along the lower edge of the diagram is shown the expected sequence of eight flowering stages through which our ideal plant passes. If it is easy to assess the relative numbers of $\mathrm{B}, \mathrm{F}$ and $\mathrm{G}$ floral shoots, then a 'BFG' score provides a concise method of recording the stage of flowering. If we are interested to know when peak or maximum flowering occurs in a plant corresponding to Fig. 1, the 'BFG' score would be ' $\mathrm{Fb}=\mathrm{g}$ ', and it might be easier to recognize the event by checking when the numbers of buds and gone-overs are equal rather than assessing when the number of open flowers is at a maximum. Similarly, 'mass flowering' could be defined as lasting from the first appearance of a gone-over to the disappearance of the last bud, knowing that this coincides with a sufficient number of open flowers to represent a good display. Alternatively mass flowering could be defined as the period when $\mathrm{F}$ is more numerous than $\mathrm{B}$ and $\mathrm{G}$, that is, all the 'BFG' scores taking the form 'Fxy'. However, the Foxglove example described below shows that this scoring method cannot be applied to all species.

Detailed measurements made on two species reveal some of the ways in which real plants deviate from our ideal example.

Fig. 2 shows the Jeffree diagrams for two individual Foxglove plants in RBGE's Demonstration Garden flowering in June and July 2007. Flowering lasted in both plants 

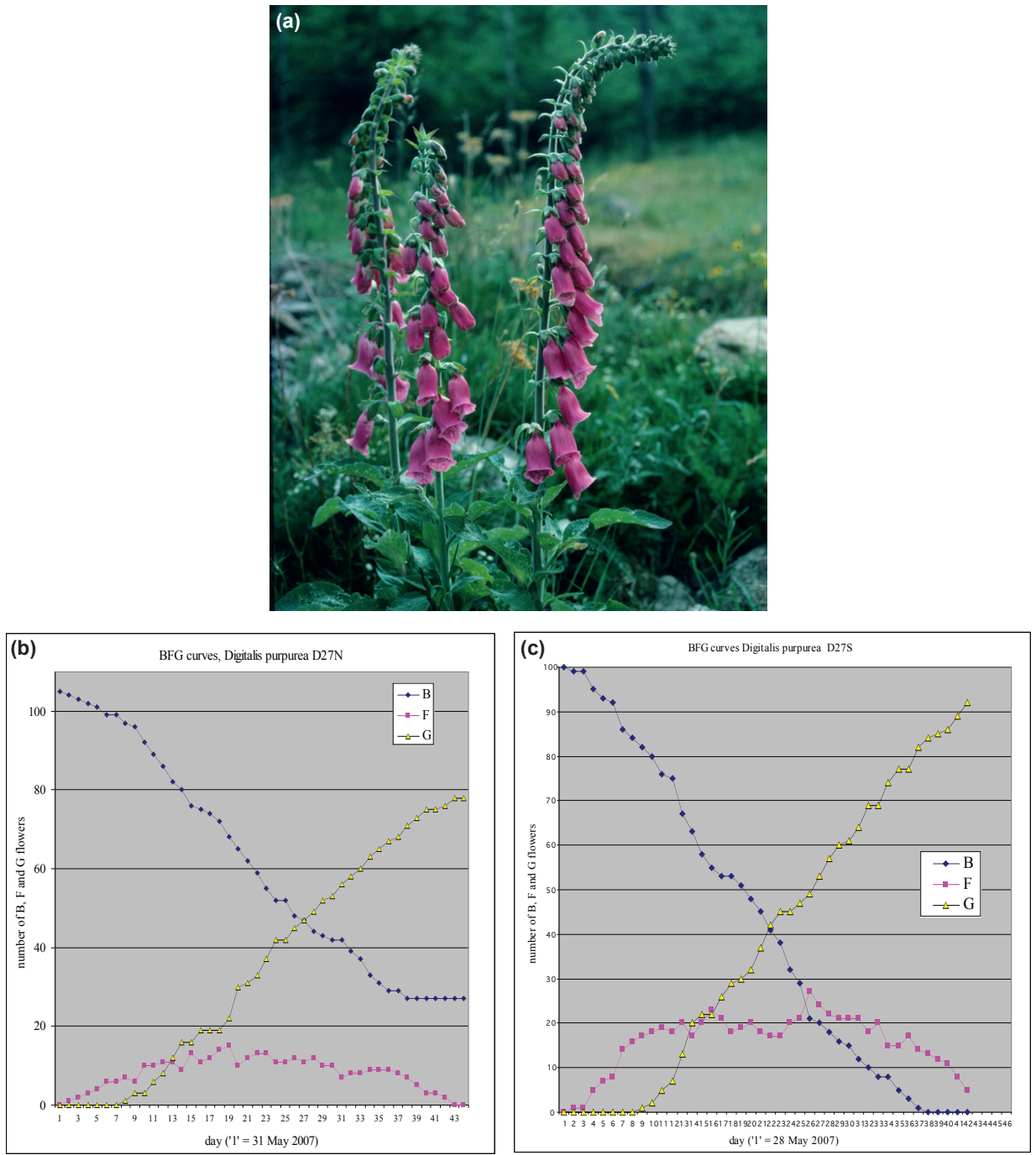

Fig. 2 (a) Foxglove Digitalis purpurea. (b) and (c) Jeffree diagrams showing daily counts of B, F and G floral shoots in two Foxglove Digitalis purpurea plants (accession number 1969.4179A in bed no. D27, May-July 2007). The vertical scale shows absolute counts. Photo: RBGE Library and Archives.

for 40-45 days, with the number of flowers open (F) at any one time rarely more than $20 \%$ of the total number (which happened to be 100 or a little more on both plants). This species is a good example of the 'sequentially flowering' type. Since most of the flowers are arranged in a more or less linear sequence up the main stem it was possible to record the state of each individual flower every day, and so measure the longevity of every flower. In the plant in bed D27S, for instance, the flowers that opened lasted on average 6.7 days (standard deviation 1.2 days) before the corolla fell off, which was the criterion used to mark the transition from $\mathrm{F}$ to $\mathrm{G}$ states. 
Although it might not mean much to talk about 'peak flowering' in a sequentially flowering species like Foxglove, it will be noticed that the maximum number of flowers does not coincide with the crossing point of the B- and G-curves. This is generally the case when the B- and G-curves have different gradients. So here is a species in which the event $\mathrm{B}=\mathrm{G}$ cannot be used as an indication of peak flowering.

Another interesting observation was that a significant number of buds did not open at all at the apex of one inflorescence. This was also seen in similar measurements made on Rosebay Willowherb, Chamerion angustifolium, in 2006. The possibility of buds not opening means that we must be cautious about using the opening of the last bud to mark the end of mass or peak flowering; but again for some species resembling the ideal
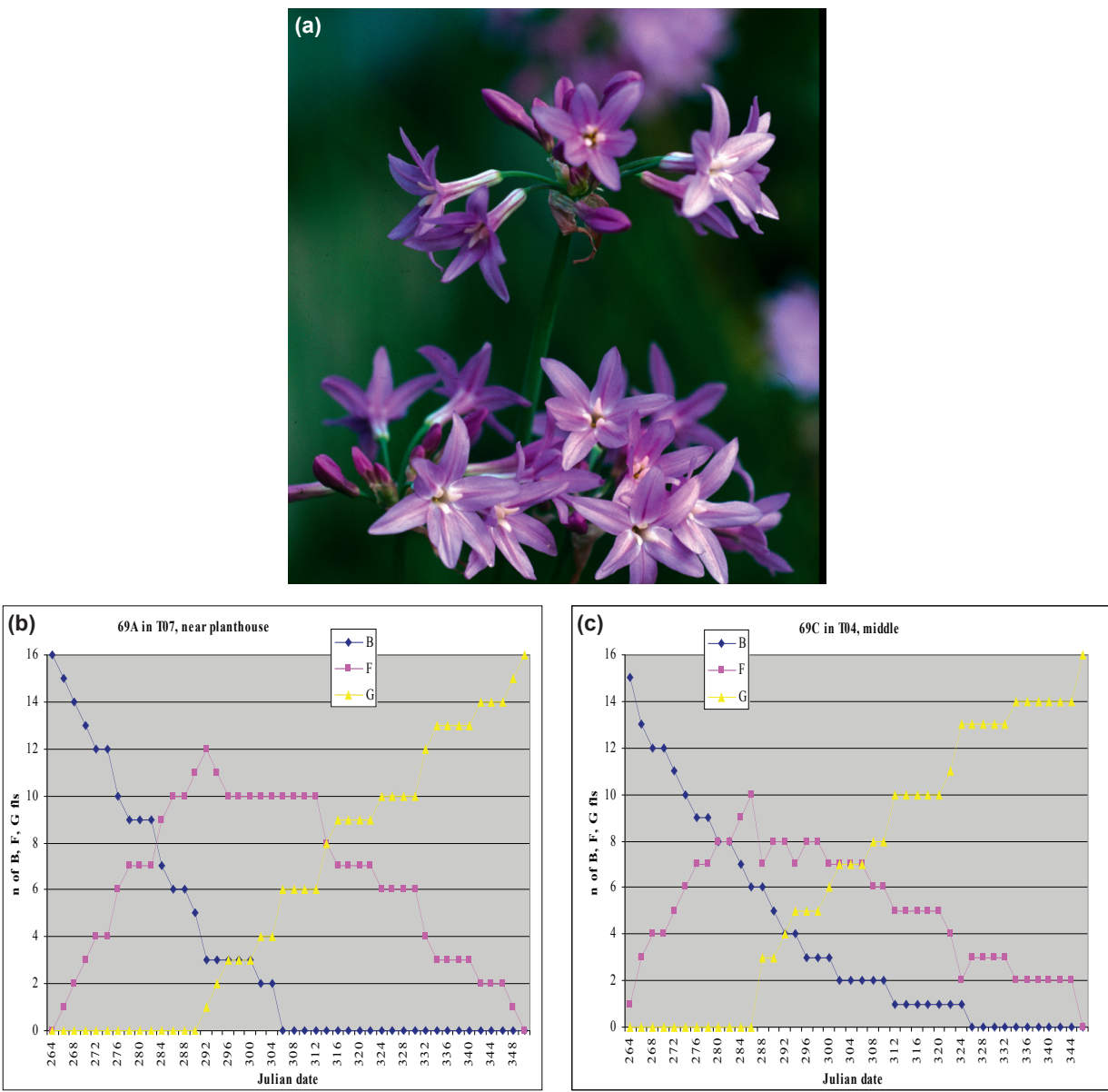

Fig. 3 Tulbaghia violacea (a) two inflorescences; (b) and (c) Jeffree diagrams showing daily counts of B, F and G floral shoots in two plants (alternate days plotted) in 2007 (left, 1974.4269 A in bed T07, right, 1974.4269 C in bed T04). The vertical scale shows absolute counts. Julian day 264 is 21 September, and 344 is 10 December. Photo: RBGE Library and Archives. 
plant in Fig. 1, in which buds only very rarely fail to open, B = 0 may indeed be a useful criterion for 'end of mass flowering'.

Other 'sequentially flowering' plants include shrubby Hypericum taxa, such as $H$. 'Hidcote', in which each flowering branch typically has about 7 flowers, only 1-2 of which are open at any one time. Fuchsia magellanica is similar: each branch may have about 20 flowers, with only up to 6 open simultaneously.

In evolutionary terms, if the 'primitive' or 'generalized' angiosperm is considered to be a perennial with continuous vegetative growth, continuous production of reproductive meristems, and continuous flowering, then 'sequentially flowering' species represent a weak specialization, with flowering occurring during a relatively prolonged flowering season in sequence along each flowering stem. Contrasted with this are the 'simultaneously flowering' plants, which have more specialized inflorescences, often in compact umbel form, producing a more concentrated display of colour in a relatively short flowering period.

Given the time constraints in preparing this article, it was not possible to use a spring-flowering species such as Hyacinth Hyacinthus orientalis or the shrub Enkianthus campanulatus, in which all the flowers on a single plant may be open simultaneously. Instead it was necessary to find an autumn-flowering species to serve as an example of the 'simultaneously flowering' type, and the most suitable turned out to be Tulbaghia violacea (Fig. 3a). It is admittedly not an ideal example, but the F-curve in the left-hand (b) diagram does reach 75\% (12 flowers open in an inflorescence of 16 flowers), which is not so different from fully simultaneous flowering. It is clear that the plant with more simultaneous flowering, in bed T07, has the shorter overlap of the B- and G-curves. A plant in which F reaches 100\% would have non-overlapping B- and G-curves.

As in the case of the Foxgloves, the F-curves are asymmetrical, associated with unequal gradients of the B- and G-curves. Moreover, the gradient of each curve decreases with time, possibly reflecting the cooling of the weather in later autumn. Both plants remained in flower for about 80 days, which would be exceptionally long for a typical simultaneously flowering plant. Interestingly, the plant in bed T07 (Fig. $3 \mathrm{~b}$, left) was situated close to pipes carrying hot water for heating the Main Range of glasshouses, while bed T04 is in unheated soil. This may explain the greater decrease in gradients of the B- and G-curves, and the greater asymmetry of the F-curve, seen in the latter plant. Even so the duration of flowering was the same in the two plants.

These examples of 'sequential' and 'simultaneous' types of flowering represent one kind of variation in flowering strategy and it is assumed that many other species would occupy an intermediate position in this dimension of variation. There are however other possible F-curve shapes. It has to be admitted that the Foxglove diagrams (Fig. 2) do not provide a complete record, since at least one of the plants produced small additional axillary inflorescences below the main spike. If the flowers on the 2-3 extra spikes had been included in the daily counts, they would have been reflected in higher $F$ values towards the end of the flowering period and the F-curves would have acquired a more irregular shape. To take another example, casual observations in the autumn and winter 
of 2007 suggest that some members of the Araliaceae, in particular Ivy Hedera helix and Fatsia japonica, would produce F-curves with two distinct peaks. In each individual flowering shoot the terminal inflorescence flowers for a week or two, and then there is a flowerless period before the subterminal inflorescences come into flower. In both species the inflorescences throughout an individual plant seem to be quite well synchronized, so the F-curve for a whole plant would consist of two separate, or almost separate, peaks. If this pattern were confirmed by daily counts on a representative selection of flowering branches on an Ivy or Fatsia plant, it would be interesting to speculate whether the species gains any selective advantage from this peculiar flowering strategy.

BFG curves reveal interesting information about flowering strategies, and suggest a number of ways in which the relative numbers of B, F and G floral shoots could be useful in rapid-survey monitoring of phenology. Quite a number of species have however been found unsuitable, for a variety of reasons. In Cistus and shrubby species of Potentilla, for example, the B and $\mathrm{G}$ states are hard to distinguish, since when a flower goes over the sepals close up once more to resemble an unopened flower. In some Daphne and Fuchsia species, and Fatsia japonica, unfertilized flowers drop off, making it difficult to estimate the relative number of G flowers. In Arabis alpina and Ulex europaeus (Gorse), it is suspected that new vegetative growth and new flower buds can develop throughout the very long flowering season (365 days in Gorse as a species, though possibly not in each individual plant). This means that the total number of floral shoots in all states cannot be assessed at the beginning of the flowering season. Gorse, Viburnum tinus, and other species also cause confusion by carrying $\mathrm{B}$ and $\mathrm{F}$ flowers of one 'flowering generation' at the same time as G flowers of the previous flowering period, which undermines the theoretical assumption of a single statistical population of floral shoots under observation through a given flowering season. In Jasminum nudiflorum, Forsythia species, and some rhododendrons, it is not easy to distinguish floral and vegetative buds, which makes assessing the number of B floral shoots difficult or impossible. Finally, in Viburnum tinus and many other plants, when some flowers in a compact inflorescence open, their petals may hide adjacent buds so that maximum display is achieved with only a proportion of the flowers open, while the hidden buds make BFG scoring difficult.

We therefore no longer use the BFG rapid-survey method for many species, but still believe that it will be useful for some. In Foxglove, for instance, the first record of a gone-over flower, which is recorded as the date the corolla falls off, for example, Bfg on day 8 in the D27N plant (Fig. 2), would be a reasonable criterion for the start of mass flowering. However, it is not easy to suggest a BFG criterion for the end of flowering in this species. It is always possible that, even in plants not suitable for the full BFG scoring, relative numbers of two of the states might still be a reliable guide to a phenological event it is useful to record. 


\section{COUNTING FLOWERS AND INFLORESCENCES}

The most objective method is simply to count the number of open flowers on a plant each week. For some plants it is easier to count inflorescences, as in the case of Asteraceae with their sometimes minute flowers but easily countable inflorescences. In this case the inflorescence is recorded as in the F state if at least one flower is open. In fact, counting inflorescences has been found to be more practicable than counting flowers in several other plants as well, such as Viburnum tinus and several rhododendrons.

It is easy enough to make a rapid and accurate count of flowers or inflorescences where there are no more than 10, but with much higher numbers it becomes more difficult and time consuming, and consistency between observers' estimates can decrease significantly. It is possible to speed up the process and improve objectivity by grouping counts into progressively larger intervals as numbers increase. We generally therefore record counts exactly up to 9 , and higher numbers are grouped as '10+' (10-14), ' $15+$ ' (15-19), '20+' (20-29), '30+' (30-39), '40+' (40-49) and '50+' (50 upwards).

Unfortunately this simple method is not useful for all accessions, mainly because on many woody plants or patches of herbs 50 flowers or inflorescences may be so far short of the number open at maximum flowering that the scale we use fails to show when maximum flowering occurs. Moreover counting flowers is tiring and time consuming, and it is unreasonable to expect volunteer observers to perform the operation on a large number of accessions.

Fig. 4 shows results obtained from Iris unguicularis from mid 2006 to week 23 of 2008. The histograms (pale grey squares) represent the counts only symbolically, since

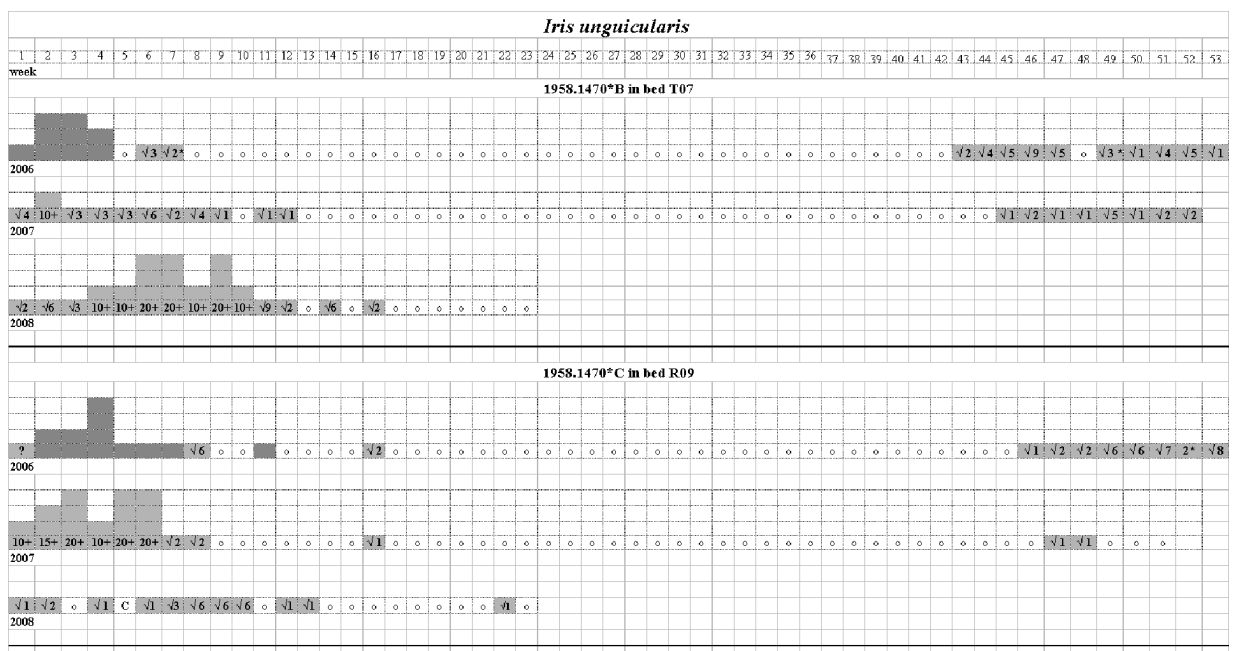

Fig. 4 Flowering scores for two patches of Iris unguicularis up to week 23 in 2008. The 'tick1-4' method was used in early 2006, and the histograms (dark grey squares) represent the '1-4' scale. In the 24 months from mid 2006, the flowers were counted as described in the text, and the histograms represent the successive steps in the scale. 
the heights of the columns are not proportional to the counts. Flowering occurs at low intensity from week 43 (late October) to early January, and peak flowering occupies the next month or two. Sporadic flowering then continues until about week 16 or even later in some years. (The mass flowering of the patch in T07 through the cold weather of March 2008 may perhaps be due to the fact that underground hot water pipes run close to the plants.) If the observations were exact counts of flowers, it would be possible to add the results of successive years to build a composite histogram showing the generalized shape of the flowering season over a period of years; unfortunately, however, this operation is not legitimate where intervals are used, since adding intervals such as ' $20+$ ' and ' $10+$ ' may give a misleading answer $(20+10=30$, or ' $30+$ ', but $29+14=43$, or ' $40+$ ').

\section{ANOTHER OBJECTIVE METHOD - PHOTOGRAPHY}

Although it has not as yet been tested, another method was suggested by $\mathrm{SH}$ as a rapid and objective method. Where the flower colour is different from the other parts of the plant, as is usually the case, it should be possible to photograph the plant digitally each week from a standard position, and then automatically measure the colour saturation of the image using an appropriate wavelength. The wavelength - the flower colour itself or a component of it - would be chosen to achieve discrimination between flower and foliage or other objects present in the image, and tests would be necessary to find the wavelength which gave the best correlation between saturation and number of flowers.

Some problems are anticipated in so far as light quality (bright sun, overcast, etc.) would be expected to vary considerably between monitoring dates, and this might affect correlation between colour saturation and flower number. The problem might be overcome by including in the image a coloured panel corresponding to the wavelengths used for the colour-saturation measurement. This would enable the colour-saturation scale on any given day to be calibrated against the standard panel.

Attractive though this idea is, and practical though it may prove to be once developed, it would involve the observer carrying a camera and also a set of colour panels to be attached to the plants before they were photographed, followed by processing of the images. This takes the method outwith our definition of 'rapid surveying'. Even so we recommend it for serious consideration in botanic garden phenology studies since it could yield quantitative data in a more intensive study of a few species, or if the botanic garden can employ a professional phenologist.

\section{‘TICK1-4’ METHOD}

Our most frustrating method - sometimes! - yet in the end the most generally useful one has been dubbed 'tick1-4'. It is based on the approach used by one of us (LR) in West African rain forest for studying the phenology of trees which serve as a food source for gorillas and other forest mammals. 
The method was based on the need for a monthly assessment of the state of the foliage, flowering, and fruiting in tree canopies at a distance, using binoculars. Counting of flowers or inflorescences, or BFG assessment, is clearly impractical for many plants in these field conditions, and the only thing one can really do is to assess the quantity of leaves, flowers or fruit as a proportion of the maximum expected. In practice this can be simplified to estimating the proportion of the canopy covered in leaves, flowers or fruit, assuming that one has a reasonable idea of what maximum coverage looks like. A scale 0-4 was used, with a tick to indicate trace presence. Adding the scores of 10 individuals gave a scale $0-40$ for each species.

Using such a method, the question arises as to how many divisions there should be between the extremes of 'no flowers' and 'maximum flowering'. Attempting great sensitivity by using many divisions, or points on the scale, would be unsatisfactory on account of inaccuracy and lack of objectivity: independent observers would be unlikely to agree on the score to represent any given tree. In general, fewer divisions recognized on such a scale mean lower sensitivity or information content, but greater accuracy and objectivity. For instance, simply recording presence or absence would be the most accurate, but least informative. Recognizing two divisions on the scale - say, up to half of maximum, and over half - would be more informative and still quite accurate. In some of the West African work a scale of four divisions was used (for example Chapman et al., 2005), and this has been adopted in the Weekly Project at RBGE, but in other studies (for example Voysey et al., 1999) the 0-4 scale was divided into 10 divisions by using a unit of 0.5 together with a tick for trace presence. (The RBGE Rhododendron Project is trialling a similar scale based on three divisions.)

For plants bearing more than 10 open flowers (or inflorescences, if these are the counting unit), ' 1 ' is recorded where there are open flowers and flowering is up to $25 \%$ of maximum, '2' for $26-50 \%$, ' 3 ' for $51-75 \%$, and ' 4 ' for $76-100 \%$.

As in the BFG method, cases where it is difficult to decide between two scores, say ' 2 ' and ' 3 ', including instances when the plant is actually at a borderline value, say at $50 \%$ of maximum flowering, are allowed for by use of transitional scores - in this case ' $2-3$ ' or ' $2 / 3$ '. We try to keep these to a minimum, however, and use them as an informal test of the consistency of the method: if half, or even just a third, of records for a particular accession takes the transitional form, it suggests that there is an unacceptably high degree of uncertainty in assigning the plant a simple score, such as '2' or ' 3 '. It would in effect be converting the 4-interval scale to a 7-interval scale, with the implications already mentioned for apparent gain in sensitivity but actual loss of accuracy.

Although a bush or tree with at most 10 flowers open would not normally be regarded as 'in bloom' from a horticultural or aesthetic point of view, recording very small numbers of flowers, sometimes for an extended period before mass flowering or even in normally flowerless times of the year, can be of considerable scientific interest. We therefore make an objective count of flowers (or inflorescences) where there are at most 10 ; so, if four flowers are open, ' $\sqrt{ } 4$ ' would be recorded to show that it is a count and not the highest interval on the ' $1-4$ ' scale. So the 'tick1-4' method combines the 
objective count up to 10 and the use otherwise of the ' $1-4$ ' scale. With a few species however counting is impractical or not useful, in which case the ' $1-4$ ' scale is used on its own.

Of course the ' $1-4$ ' scale is not objective in the same sense as counting. One problem is that different observers may not have the same idea of what '100\% flowering' means, and it may be difficult even for one observer to keep in mind, over a number of years, the same image of $100 \%$ flowering for any given plant. One possible way around this difficulty would be to carry photographs - as prints, or stored in a digital camera - to show what '100\%' means for each monitored accession. Even better would be to have four images for each accession, showing $25 \%, 50 \%, 75 \%$ and $100 \%$ flowering. While carrying a bundle of printed photographs would be somewhat cumbersome, use of a digital camera might be compatible with the rapid-survey concept.

A possible substitute for a photograph is a short verbal description, which could be incorporated into the recording form or, in practice, is included for a few species in the Instructions for Observers. In the case of a truly simultaneously flowering species, ' $100 \%$ ' can be described simply as 'all fls F'. This never happens however in sequentially flowering species, and in this case a more elaborate description is required. Not only is the whole inflorescence not in flower at any one time, the different inflorescences or flowering branches on one plant may not be synchronized. For example, our provisional description of ' $100 \%$ flowering' in Forsythia $\times$ intermedia is '2 flowers open on each of a majority of flowering branches'; but a problem with this is that, as in the case of Jasminum nudiflorum, it may not be clear which buds are going to produce flowers and therefore which branches are potentially flowering ones. For Fuchsia magellanica ' $100 \%$ flowering' means ' 4 flowers open on each of two thirds of flowering branches', and in this case it usually is clear which branches are (going to be) flowering, since the pendant flower buds are distinctive.

The species of plant most likely to cause a nervous breakdown among phenology monitors in our project is without doubt Mahonia $\times$ media. This is a sequentially flowering plant, with at most half of the flowers on any one spike open at once. The spikes constituting an inflorescence at the tip of a branch are usually unsynchronized, and the inflorescences on a bush are also unsynchronized. Moreover the buds can be almost as bright yellow as the open flowers (which might cause problems for the photographic method). Our provisional description of ' $100 \%$ flowering' is ' $50 \%$ of flowers open on two thirds of spikes', but that does not seem to make it any easier to assess a plant on the ' $1-4$ ' scale when it is well short of maximum flowering or is going over.

A further problem is that maximum flowering of an accession in one year may not be much like what it achieves in another year. This may be due to an intrinsic rhythm in the plant, or to environmental conditions in the year of flowering or the previous season. Flowering performance may also be affected by disease or other decline in the plant's condition, or of course an actively growing tree or expanding patch of herbs may be expected to increase its number of flowers significantly every year. In using the ' $1-4$ ' method it would need to be decided therefore whether ' $100 \%$ flowering' is meant to 
represent the maximum achieved in the year in question; but it will often be impossible to predict this in advance of peak flowering. On the other hand ' $100 \%$ ' could represent the maximum in an average year, or alternatively the maximum over a decade or longer period. In all these cases the question arises as to how to record maximum flowering in a year with exceptionally good flowering. One possible solution would be to use a ' 5 ' to record flowering 'greater than $100 \%$ '.

We need not lose sleep over these theoretical problems if it is accepted that the ' $1-4$ ' method is not and does not pretend to be objective. At the same time it remains a very useful method - and often the only one available in rapid surveying. In fact there is no point in worrying too much about the exact meaning of ' $100 \%$ ' so long as the score goes up when the number of flowers increases significantly from week to week, and goes down when the number of flowers is declining. This approach recommended itself when a new species was added to the project in December 2007: Pyracantha crenulata had a small number of inflorescences in bud, with a few flowers open and a few gone over, but none of the monitors had ever seen it fully in flower, so none of us had much idea what ' $100 \%$ flowering' should correspond to. In this case it makes sense, assuming that it will at least have many more flowers at some other season, to give it a score of ' 1 ', and then increase the score as more flowers appear. The actual score reached, whether '4' or less, is probably not reliable and so does not contain much useful information; but the change from week to week - whether positive, negative or zero - does convey useful information, so that a histogram plotted from the results should give a good idea of when peak flowering occurs and how rapidly flowering builds up to the peak and then drops off.

A verbal description or photograph can help maintain consistency of recording, but equally important is the quality of the observers and the way the monitoring is organized. A total of 214 accessions belonging to 117 taxa (mostly species) are monitored each week, all year round, in our Weekly Project at RBGE. The route is divided into southern and northern sections, the former beginning at the East Gate and ending conveniently at our watering hole (the Terrace Café beside Inverleith House), where monitors on the Weekly and Rhododendron Projects often meet up for coffee and a chat. The northern route then proceeds via the Demonstration Garden to the beds near the glasshouses and Herbarium before terminating at the East Gate. Normally each section of the route is monitored by two observers, which is an enjoyable and easy way to complete the task (in about an hour), using a certain division of labour, but if someone is away it is quite possible for one observer to complete a half-route. When several people are away, one team of two can complete both routes, and in emergency it is possible for one person to do all the monitoring single-handed, as happens occasionally.

With this flexible arrangement it is rarely if ever that a week's monitoring is missed, and we feel that it is important in this kind of project to maintain a continuous record. It is also important that all observers remain in regular contact with all the monitored plants - partly for the practical reason that sometimes labels are lost, the monitored plants change through growth, and neighbouring plants grow and perhaps hide the 
monitored plants, meaning that it may sometimes be difficult to know which plant is to be recorded.

In addition, a single species may be growing in different conditions in different parts of the route - such as Geranium robertianum on the southern section, in open conditions and with very long flowering and foliage seasons (it is evergreen), while on the northern section it grows under trees, with a shorter flowering season and sometimes no leaves showing in winter. In this kind of situation, regular observation by every monitor of the same species growing in different conditions may suggest ideas to explain the phenological behaviour of different species, and even differences between populations of the same species. Accordingly the two teams of observers regularly swop half-routes so that everyone regularly sees every plant in the project.

So, although strict scientific objectivity cannot be claimed for the 'tick1-4' method, it can be made reliable enough by using the same team of competent observers over many years. As their knowledge of the plants accumulates, and as they get used to the method and their experience grows their consistency of observation increases. The information collected may not be acceptable for publication as scientific data, but it should reveal major changes in flowering phenology reliably enough, and help to identify taxa that merit more intensive investigation using objective methods.

\section{COMPARISON OF METHODS}

After the first three years of the Weekly Project we have concluded the preliminary exploration of monitoring methods, and believe that all three discussed in this article have a useful role. No one method is appropriate for all species, and we are not in a position to give detailed recommendations for individual taxa. A few general rules emerge, however, which may be of use to phenologists setting up a similar project.

One simple objective method is simply to record presence or absence of open flowers. Another is the counting of flowers or inflorescences. These methods are recommended if observations are intended for scientific analysis. Both require a clear criterion for identifying the transitions $\mathrm{B}$ to $\mathrm{F}$ and $\mathrm{F}$ to $\mathrm{G}$. Counting also requires (a) an unambiguous counting unit, whether flower or inflorescence (usually not a problem); (b) good access to the whole plant, or to a clearly defined part of the plant (or patch of herbs), so that a complete count can be made; and (c) that the maximum expected number of flowers or inflorescences is countable within the time available. The counting scale can be absolute numbers throughout, recommended where practical, or using grouped numbers for high frequencies if this improves accuracy. If counting is impractical, just recording presence/ absence of open flowers can yield useful information (see Harper \& Morris, 2007).

The BFG method can be useful for providing markers for start and end of mass flowering, and possibly also for peak flowering where the Jeffree diagram resembles that for an ideal plant (Fig. 1). However, some experience needs to be gained first in order to check that the marker, for instance first appearance of a G-state flower, does in fact coincide with the phenological event it is intended to mark, in this case start of 


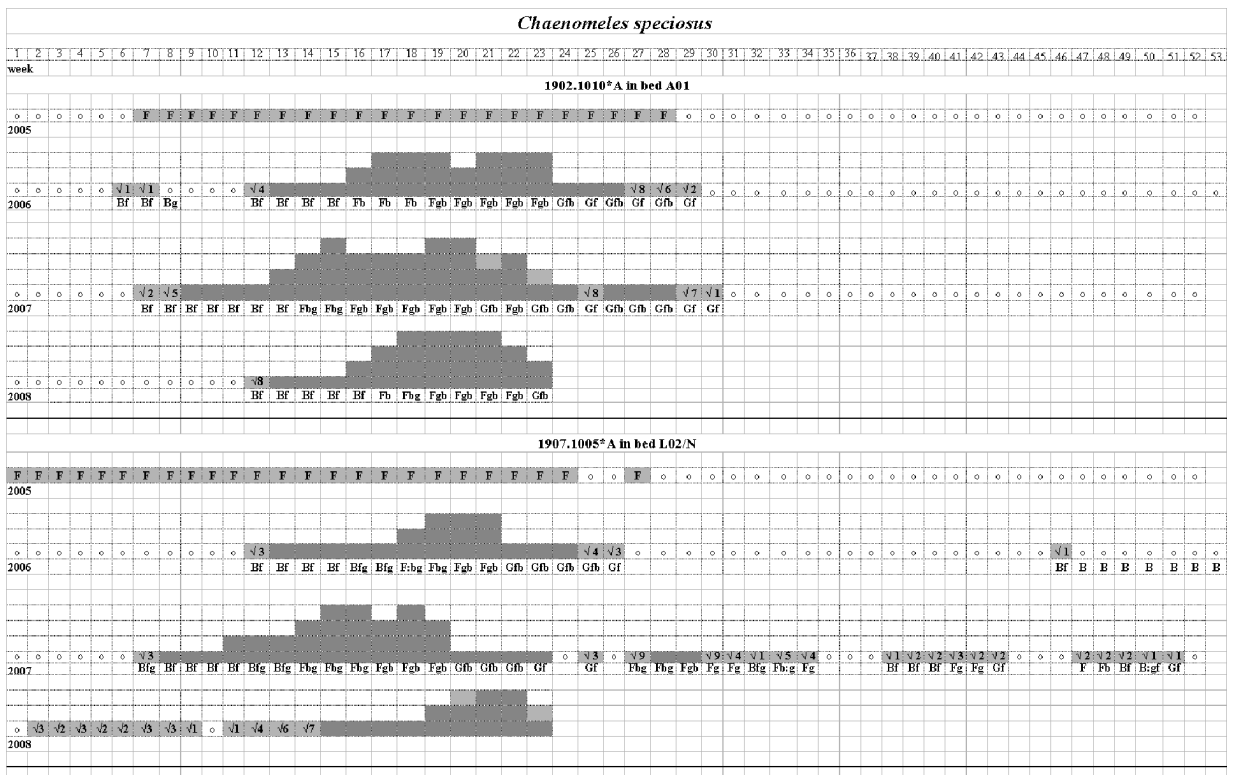

Fig. 5 Flowering scores for two bushes of Chaenomeles speciosus up to week 23 in 2008. Only presence/ absence of flower was recorded in 2005. The 'tick1-4' and 'BFG' methods were used in 2006-8. A pale grey square on top of a dark grey one indicates a transitional score, such as ' $2-3$ '.

mass flowering. There is no general rule, applicable to all plants, enabling BFG stages to be linked reliably with quantitative characteristics such as start and end of mass flowering.

The least objective but generally most useful method for rapid surveying is 'tick1-4'. The quality of information gathered depends to a considerable degree on intangibles such as the expertise, dedication and teamwork of the observers. Given an awareness of its limitations from a scientific point of view, we believe this method is legitimate. It has the advantage of using objective counts for small numbers of flowers, and these can incidentally provide a reliable criterion for start and end of mass flowering for many plants - especially where there is a prolonged period before or after mass flowering when there are only few flowers open. For each species a decision can be made on the minimum number of open flowers (or inflorescences) constituting mass flowering, and if it is practical to count up to that number then the start and end of mass flowering can be fixed objectively. Although 'tick1-4' can be attempted on most species, some prove to be difficult, such as Mahonia $\times$ media, and the results may not be sufficiently reliable.

Some of these points can be illustrated by Chaenomeles speciosus (Fig. 5). In 2005 flowers were recorded as merely present or absent, which soon proved to be inadequate, especially considering that the species turned out to be capable of flowering virtually all year round. It was replaced by the 'BFG' and 'tick1-4' methods in 2006. The latter method probably gives a reasonably accurate idea of when mass flowering occurs. If mass flowering is taken to be the presence of at least 10 open flowers, it can be seen 
that the late springs of 2006 and 2008 are reflected in the late date of the first dark grey square in both accessions. If a higher threshold is required for mass flowering, then it is likely in this species that the 'BFG' scores beginning with ' $\mathrm{F}$ ' are a fairly good guide - in other words scores in which open flowers are more abundant than both buds and gone-over flowers. This could however be misleading in the case of weeks 27-31 and later in 2007, which are short periods considered to be flowering periods distinct from the main spring flowering, and involving rather small numbers of flowers.

We have also tried a few supplementary methods. One of these is an 'aesthetic score', on a 1-4 scale, in cases like Crocosmia pottsii and Daphne bholua, where the buds and/or gone-over flowers can make a significant contribution to the aesthetic appeal. In these cases, scoring for the number of flowers may not give an accurate idea of when a plant looks at its best. Other characteristics could also be scored separately, such as scent.

\section{ACKNOWLEDGEMENTS}

We tender our thanks to Christine and Roy Thompson for discussion of methods and for monitoring on 27 and 28 October 2007; to RT and Chris Jeffree for directing our attention to the Jeffree diagrams; and to the RBGE Rangers for looking after the Phenology Envelope in the East Gate kiosk every week, and for providing the lexicographic gem.

\section{REFERENCES}

CHAPMAN, C.A., CHAPMAN, L.J., STRUHSAKER, T.T., ZANNE, A.E., CLARK, C.J. \& POULSEN, J.R. (2005). A long-term evaluation of fruiting phenology: importance of climate change. Journal of Tropical Ecology, 21, 1-14.

GLOVER, B.J. (2007). Understanding Flowers and Flowering. Oxford: Oxford University Press.

HARPER, G.H., MANN, D.G. \& THOMPSON, R. (2004). Phenological monitoring at Royal Botanic Garden Edinburgh. Sibbaldia, 2, 33-45.

HARPER, G.H. \& MORRIS, L.S. (2007). Flowering and climate change - part II. Sibbaldia, 5, $25-42$.

JEFFREE, E.P. (1951). A modified phenological approach in the determination of flowering times of beekeeping (and other) plants in Aberdeenshire - Proc. XIVth International Beekeeping Congress, Leamington Spa, Sept. 1951, 1-6.

JEFFREE, E.P. (1957). Some aspects of the seasonal course of honeybee colonies and the changing background of plants on which they forage. DSc Thesis, University of Aberdeen, December 1957.

VOYSEY, B.C., McDONALD, K.E., ELIZABETH ROGERS, M., TUTIN, C.E.G. \& PARNELL, R.J. (1999). Gorillas and seed dispersal in the Lopé Reserve, Gabon. I: Gorilla acquisition by trees. Journal of Tropical Ecology, 15, 23-38. 
